\title{
The Evolution of Sex Chromosomes and Sex Determination in Vertebrates and the Key Role of DMRT1
}

\author{
M. Ferguson-Smith \\ Cambridge Resource Centre for Comparative Genomics, Centre for Veterinary Science, University of Cambridge, \\ Cambridge, UK
}

\section{Key Words \\ $D M R T 1$ - Sex determination - Sex differentiation - SRY .}

Vertebrates

\begin{abstract}
Mechanisms of vertebrate sex determination are reviewed in the light of what is known about the origin and evolution of sex chromosomes. All vertebrate males have testes that are similar in anatomy and in spermatogenesis. Despite a variety of sex chromosome systems, the same cascade of genes operate in the differentiation of testes and male genitalia. Only the primary signal or switch that initiates the cascade may be different. Genetic sex determination (GSD) occurs in most species, although temperature-dependent sex determination (TSD) is the switch in some reptiles without GSD. SRY is the genetic switch in eutherians (placental mammals) and DMRT1 may have that function in birds and at least one species of fish. The switch in all other groups of vertebrates with either male or female heterogamety is unknown. DMRT1 is an ancient sex determining gene, found first in invertebrates, and is one of several genes expressed in higher levels in the vertebrate embryonic testis than in the embryonic ovary. It
\end{abstract}

Studies on comparative genomics and evolution were supported by a grant to M.F.S. from the Wellcome Trust.

\section{KARGER}

Fax +4161306 1234

E-Mail karger@karger.ch

www.karger.com (c) 2007 S. Karger AG, Basel

1661-5425/07/0011-0002\$23.50/0

Accessible online at:

www.karger.com/sxd is singled out in this review because of its likely key role in sex determination both in birds and in reptiles with TSD. DMRT1 is one of the few sex genes that have been mapped in representative species of fish, turtles, crocodiles, amphibians, birds and mammals. Greater effort in comparative mapping, sequencing and expression studies is required to discover the other primary switch genes in vertebrates and to answer questions about their evolution.

Copyright $\odot 2007$ S. Karger AG, Basel

Our present knowledge of mammalian sex determination is based on studies over 45 years ago, of Klinefelter and Turner syndromes in humans and mice that revealed the dominant $\mathrm{Y}$ chromosome factor in male differentiation. Studies in XX males led to the discovery of $S R Y$ as the primary testis determining factor (TDF) and many of the other known factors in the sex determination cascade (table 1) have been characterised by the careful investigation of sex-reversed XY females. The work was initiated largely by pathologists and clinicians who studied patients referred to them with disorders of infertility or sex differentiation. The solution of these problems has required the collaboration and expertise of geneticists and molecular biologists. Disorders of sex determination are not lethal and so provide an excellent model for the study of biological systems. 
Introduction to this fascinating field was initially through the study of numerical and structural abnormalities of the human sex chromosomes, prompted by the paradoxical nuclear sex chromatin findings that were widely thought to indicate that Turner and Klinefelter syndromes were sex reversed males and females respectively. An early chromatin-positive Klinefelter patient was observed to have XY sex bivalents and sperm in a single seminiferous tubule (Ferguson-Smith and Munro, 1958) suggesting that he was not sex reversed, and that the answer was to be found by karyotype analysis. The 47,XXY and 45,X karyotypes in Klinefelter and Turner syndromes were reported in 1959 (Ford et al., 1959; Jacobs and Strong, 1959), and the location of TDF on the short arm of the Y was shown later (Jacobs and Ross, 1966). The anomalous segregation of the Xg sex-linked blood group in families of XX males led in 1966 to the hypothesis that there had been an interchange between $\mathrm{Xg}$ on the X and TDF on the Y (Ferguson-Smith, 1966) and to the discovery of $S R Y$ much later (Sinclair et al., 1990). In all these cases, human disorders led to an understanding of normal processes, and there are many more examples in the field of human sex determination.

It is apparent that all vertebrates share with humans the same cascade of genes responsible for testis differentiation, spermatogenesis, and the development of male internal and external genitalia. However, the primary switch $(S R Y)$ that initiates this cascade in humans, and most mammals, is different in other vertebrates. It has become clear that this difference has arisen from the occurrence of separate sex chromosome systems that have evolved independently from one another. It seems an appropriate time to consider what is known about the evolution of sex chromosome systems and the different mechanisms for initiating testis differentiation.

\section{Evolution of Vertebrate Sex Chromosomes}

Studies on the evolution of sex chromosome systems have been based during the last 40 years on the hypothesis of Susumu Ohno (1967) who observed the variable size of the $\mathrm{W}$ chromosome and the invariable size of the $\mathrm{Z}$ in different snake families. He concluded that the $\mathrm{W}$ and $\mathrm{Z}$ must have evolved and differentiated from an ancestral pair of chromosomes. The same reasoning suggested that mammalian $\mathrm{X}$ and $\mathrm{Y}$ sex chromosomes must have evolved from an identical ancestral pair of homomorphic chromosomes, one of which developed a sex determining role. The pair gradually differentiated from
Table 1. Genes involved in mammalian sex differentiation

\begin{tabular}{|c|c|}
\hline Gene & Role \\
\hline$S R Y$ & Primary testis determining factor \\
\hline SOX9 & Main target for SRY \\
\hline$D A X 1$ & Antagonist to SRY \\
\hline SF1, WT1, GATA 4 & Required by SRY \\
\hline AMH & Activated by SOX9 \\
\hline WNT4, WNT7 & Required by $\mathrm{AMH}$ \\
\hline$D M R T 1$ & Required by Sertoli and germ cells \\
\hline$A T R X$ & Required for testis development \\
\hline LHX9, M33 & Required for gonadal development \\
\hline FGF9 & $\begin{array}{l}\text { Required for peritubular myloid cell } \\
\text { migration }\end{array}$ \\
\hline$T D A 1,2,3$ & Promote ovarian development \\
\hline$A R(D H T R)$ & $\begin{array}{l}\text { Required for development of male } \\
\text { genitalia }\end{array}$ \\
\hline PAX2, LIM1, EMX2 & $\begin{array}{l}\text { Required for Wolffian and Mullerian } \\
\text { ducts }\end{array}$ \\
\hline INSL3 & Required for testis descent \\
\hline $\mathrm{DHH}$ & Required for spermatogonial division \\
\hline
\end{tabular}

one another, with the sex determining region on the $\mathrm{Y}$ becoming isolated during paternal meiosis so that recombination with the $\mathrm{X}$ chromosome was prevented. Attrition of the $\mathrm{Y}$ and accumulation of sequences involved in male gametogenesis led to a distinctive $\mathrm{Y}$ chromosome in which only small terminal parts were homologous with the $\mathrm{X}$. These parts are necessary for synapsis and segregation of the $\mathrm{X}$ and $\mathrm{Y}$ chromosomes during male meiosis. The $\mathrm{X}$ chromosomes are able to pair and to recombine with one another throughout their length in female meiosis but, in male meiosis, can only undergo recombination with the $\mathrm{Y}$ at the small pairing (pseudoautosomal) regions, leaving a large differential segment containing the strictly X-linked genes. The similar dosage of Xlinked genes in males and females is explained satisfactorily by $\mathrm{X}$-inactivation, whereby the genes in one of the two X chromosomes in the female were silenced (Lyon, 1961). The conservation in form and gene content of $X$ chromosomes in all mammalian species is explained by the two processes of meiotic isolation and $\mathrm{X}$-inactivation. The fact that the extant human $\mathrm{X}$ and $\mathrm{Y}$ share a small number of genes active on both their pairing and differential segments, that lead to the haploinsufficiency in Turner syndrome (Ferguson-Smith, 1965), attest to their origin from an ancestral pair. Rearrangements between the $\mathrm{X}$ and autosomes are deleterious as they disrupt the dosage compensation mechanism; any such change would be eliminated by natural selection. 
Ohno (1967) also proposed that the Z and W chromosomes in birds have evolved from an ancestral pair of chromosomes different from the mammalian ancestral sex chromosomes. Like the conservation of the $\mathrm{X}$ among mammals, the $Z$ chromosome has been conserved among birds. It is now known that genes on the chicken $\mathrm{Z}$ chromosome are homologous to genes on human chromosomes 9, 5, 8 and 18 (Nanda et al., 2000). Genes homologous to the mammalian $\mathrm{X}$ are located on chicken chromosomes 1,4 and 12 .

Nearly 40 years later the basis of Ohno's hypothesis still stands, although it now requires some modification.

(1) The marsupial X chromosome differs from the eutherian $\mathrm{X}$ chromosome in that genes homologous with the short arm of the human $\mathrm{X}$ are autosomal and do not require dosage compensation (Spencer et al., 1991).

(2) The chloride channel gene (CLCN4) is X-linked and X-inactivated in humans and Mus spretus but maps to chromosome 7 in Mus musculus (Palmer et al., 1995). FISH mapping reveals copies of this gene on both sex chromosomes of M. spretus (unpublished). The steroid sulphatase gene (STS) is X-linked in humans and pseudoautosomal in mice (Keitges et al., 1985); the gene escapes inactivation in both. Apart from CLCN4, genes X-linked in one placental mammal are found to be X-linked in all.

In contrast to the mammalian $\mathrm{X}$ chromosome, the mammalian $\mathrm{Y}$ chromosome shows considerable variation between species while retaining $S R Y$ (which shares homology with SOX3 on the X) and genes necessary for male fertility. Thus Y chromosome paint probes (explained in Ferguson-Smith, 1997) from one species seldom show much homology when cross-hybridised with the $\mathrm{Y}$ from other species. Within species, Y paints hybridise to the pseudoautosomal regions of the $\mathrm{X}$ and, in the human at least, to a chromosomal block on the long arm of the $\mathrm{X}$, encompassing the $\mathrm{X}$-inactivation centre.

The evolution of the human sex chromosomes has been investigated by comparing the map positions of 19 $\mathrm{X}-\mathrm{Y}$ gene pairs located on the differential segments of the $X$ and $Y$ chromosomes (Lahn and Page, 1999). The X-Y gene pairs were further characterised by determining the synonymous nucleotide divergence between the X-linked and Y-linked coding regions, which provides a measure of the evolutionary time that has elapsed since the gene pairs started differentiating into distinct $\mathrm{X}$ and $\mathrm{Y}$ forms. At that time, recombination between the $\mathrm{X}$ and $\mathrm{Y}$ homologues would have ceased due to the meiotic isolation of the differential segments. It was found that the $19 \mathrm{X}-\mathrm{Y}$ pairs could be grouped into four 'strata' in time depending on the extent of sequence divergence, each stratum having a comparatively distinct range of values. The genes in each stratum were linked on the $\mathrm{X}$ chromosome map, but were somewhat scrambled on the Y map, presumably by the occurrence of inversions in the $\mathrm{Y}$ during evolution. The oldest group, with the greatest sequence divergence, was designated stratum 1 , with the corresponding genes mapping to the long arm of the human X. Strata 2, 3 and 4 (most distal) mapped to the short arm of the $\mathrm{X}$ and were progressively younger in origin. Theoretically, it would be expected that the oldest stratum would have the fewest remaining $\mathrm{Y}$ homologues and, conversely, that the youngest would have the highest density of $\mathrm{X}$ genes with active $\mathrm{Y}$ homologues that escape $\mathrm{X}$-inactivation; these expectations are all fulfilled.

It was postulated that the results indicated that X-Y recombination was suppressed regionally in four steps during evolution; each step separated by a time interval and initiated by an inversion event on the $\mathrm{Y}$ chromosome that led to suppression of recombination and the onset of differentiation between the X-Y pairs of genes. As the $\mathrm{Y}$ genes decayed and lost their function, $\mathrm{X}$ homologues were first up-regulated for dosage compensation in the male, and later by the evolution of X-inactivation (Reik and Lewis, 2005). Lahn and Page (1999) used comparative genomics to estimate the likely timing of the origin of differentiation in each stratum. In stratum 4 (the most recent), differentiation was estimated to have arisen $30-$ 50 million years ago (Mya), in stratum 3 at 80-130 Mya, in stratum 2 at 130-170 Mya and in stratum 1 at 240 350 Mya. The differentiation of stratum 1 would have occurred shortly after the mammalian and avian lineages diverged at about $310 \mathrm{Mya}$, and of stratum 2 prior to the divergence of the monotreme lineage at 210 Mya. The fourth stratum probably differentiated during early primate evolution at the time the simian and prosimian lineages diverged.

Independent comparative studies on vertebrate species that diverged from the eutherian lineage early in evolutionary history, support the idea of a stepwise evolution of the mammalian sex chromosomes. Gene mapping in marsupials and monotremes shows that genes that currently map to the short arm of the human X are autosomal and escape inactivation in marsupials and, for example, are located in chromosomes 5 and 1 in the tammar wallaby (Graves and Westerman, 2002). The X-linked genes on the long arm and proximal short arm of the human $\mathrm{X}$ are conserved in the marsupial $\mathrm{X}$. The genes on the short arm of the human $\mathrm{X}$ and on its two pseudoautosomal re- 
gions must have been added later in the eutherian lineage by translocation from autosomes, and this is confirmed by mapping mammalian $\mathrm{X}$ genes in the platypus as in that species these genes are also autosomal (table 2). Hybridisation of X chromosome-specific DNA from the tammar wallaby to human metaphases confirms the location of the conserved $X$ genes to the long arm and proximal short arm of the human X (Glas et al., 1999). (It is noteworthy that the marsupial $\mathrm{X}$ is the only marsupial chromosome that can be successfully hybridised to human chromosomes, underlying the remarkable conservation of the $\mathrm{X}$ as postulated by Ohno). These comparative studies strongly support the idea of X chromosome strata with the conserved $\mathrm{X}$ genes corresponding to stratum 1 , and groups of autosomal genes corresponding to strata 2 and 3 added to the $\mathrm{X}$ by successive translocations. The pseudoautosomal genes seem to be later additions.

Moving from humans and mammals to birds, reptiles and fish, more is revealed about the evolution of sex chromosomes from comparative mapping in these groups. The publication of the draft genome sequence of the chicken (Gallus domesticus) in particular has enabled valuable conclusions to be drawn from comparisons with the map of the mammalian X. Kohn et al. (2004) show that stratum 1, the $\mathrm{X}$ conserved region, is derived from chicken chromosome 4 with the exception of a small 1.4-Mb region, corresponding to human Xq28, that shares homology with several chicken microchromosomes including chromosome 12 . The added $\mathrm{X}$ genes are located on chicken chromosome 12 (stratum 2) and 1 (stratum 3 ). The findings convincingly confirm the three genome regions that are ancestral to birds and mammals and that have become incorporated into the mammalian X chromosome in a stepwise fashion at critical times of divergence in vertebrate evolution. When the comparative mapping was extended to teleost fish (zebrafish, Danio rerio, and pufferfish, Tetraodon nigroviridis), Kohn et al. found that genes from strata 1 and 3 were together on several small chromosomes of both species, suggesting that they might have been present in a single chromosome in a teleost ancestor. Following a series of inversions and fission events the single chromosome became separated into several smaller chromosomes. By the time tetrapods and fish diverged 450 million years ago, strata 1 , 2 and 3 may each have been on separate chromosomes, with fusion subsequently of strata 1 and 2 prior to the divergence of marsupials and monotremes estimated at $210 \mathrm{Mya}$, and fusion of stratum 3 to 1 and 2 in the eutherian $\mathrm{X}$ about 180 Mya. These timings differ somewhat from earlier estimates by Lahn and Page (1999), and the
Table 2. Location of human X-linked genes in platypus (Ornithorhynchus anatinus, OAN) (modified from Waters et al., 2005)

\begin{tabular}{|c|c|c|c|c|c|}
\hline Human & $\mathrm{OANX}_{1}$ & Location & OAN1 & OAN2 & OAN6 \\
\hline Xp & $\begin{array}{l}A L A S 2 \\
G A T A 1 \\
U B E 1^{*}\end{array}$ & $\begin{array}{l}\mathrm{P} \\
\mathrm{P} \\
\mathrm{P}\end{array}$ & $\begin{array}{l}C Y B B \\
Z F X \\
A M E L \\
D M D \\
T I M P\end{array}$ & $\begin{array}{l}\text { OTC } \\
\text { SYN1 } \\
\text { POLA } \\
\text { MAOA }\end{array}$ & \\
\hline $\mathrm{Xq}$ & $\begin{array}{l}F 8 \\
F 9 \\
P L P \\
G L A \\
R C P \\
A R^{*} \\
G D X \\
M C F 2 \\
G 6 P D^{*}\end{array}$ & $\begin{array}{l}\mathrm{P} \\
\mathrm{P} \\
\mathrm{P} \\
\mathrm{P} \\
\mathrm{P} \\
\mathrm{D} \\
\mathrm{D} \\
\mathrm{D} \\
\mathrm{D}\end{array}$ & & $B G N$ & $\begin{array}{l}A T R X \\
R B M X \\
C D X 4 \\
\text { SLC16A2 } \\
\text { SYBL1 }\end{array}$ \\
\hline
\end{tabular}

* Gene expression equal in both sexes.

$\mathrm{P}=$ Present on pairing (pseudoautosomal) segment, not requiring dosage compensation; $\mathrm{D}=$ present on differential segment, requiring dosage compensation.

authors note that proof of this mechanism must await the comparative mapping in an outgroup species, such as shark, lamprey or amphioxus.

\section{Sex Determination in Mammals}

While $S R Y$ is the primary switch for testis differentiation in eutherians, the mechanism remains unclear. One of its functions is to activate SOX9 and thus the development of Sertoli cells. SRY is absent in some mole voles such as Ellobius lutescens, which has an XO sex chromosome complement in both sexes, and a related species $E$. tancrei, which has an XX complement in both sexes (Just et al., 1995). The spiny rat, Tokudai osimensis, also has an $\mathrm{XO}$ complement in both sexes, and the single $\mathrm{X}$ has a small region with homology to the mouse $Y$ but without SRY (Arakawa et al., 2002). These rodents have evolved a system of sex differentiation that no longer needs a Y chromosome or SRY. The mechanism that has replaced $S R Y$ remains to be determined. A similar unexplained anomaly is observed in those sterile Y-negative human sex-reversed males with an apparently normal 46,XX karyotype (Ferguson-Smith and Affara, 1988). Apart from the above exceptions, in mammals the male (XY) is the heterogametic sex. 


\section{Sex Determination in Birds}

As discussed above, comparative mapping has shown that the XX/XY sex chromosome system in mammals and the ZZ/ZW system in birds have evolved independently from two different ancestral pairs of chromosomes. In birds the female (ZW) is the heterogametic sex and $S R Y$ is absent. The $\mathrm{W}$ chromosome in most birds has undergone a similar type of attrition as the mammalian Y. However, the primitive ratite birds have a $\mathrm{W}$ almost equal in size to the $Z$ (Shetty et al., 1999) indicating that this could have been a more ancient form from which the others evolved. Unlike sex chromosome aneuploidy in mammals and in Drosophila, ZO and non-mosaic ZZW diploid birds are unknown (and probably embryo lethal), so that a sex-determining mechanism by a female-determining gene on the $\mathrm{W}$ cannot be distinguished from a dosage-dependent gene for testis determination on the $Z$ (Graves, 2003). However, triploid ZZZ are viable but sterile males and triploid ZZW chickens develop as intersexes (see below). In mammals, the exceptional XO (female) and XXY (male) individuals revealed the dominant male determining role of the $\mathrm{Y}$, whereas in Drosophila the observation that XXY flies are female and XO flies male led to the conclusion that X gene dosage determines sex in this species. Both theories of sex determination have been studied extensively in birds.

\section{Dosage-Dependent Male Determination}

The best candidate is the gene DMRT1 that maps to the $\mathrm{Z}$ chromosome (Nanda et al., 1999) and has a role in the sex differentiation pathway in many different species. The gene has homology to the doublesex $(d s x)$ gene in Drosophila and to the mab-3 gene in Caenorhabditis elegans (Raymond et al., 1998). DMRT1 maps to the short arm of chromosome 9 in humans and haploinsufficiency is thought to be responsible for the sex reversal in XY females associated with deletions in the distal end of chromosome 9p (Calvari et al., 2000). The gene is expressed in the gonads of chicken embryos, with higher levels of expression in ZZ males than in ZW females prior to and during the critical period of sex differentiation when the undifferentiated gonad first begins to form tubules (Raymond et al., 1999). Thus a double dose of the DMRT1 gene is associated with male sex and a single dose with female sex. In the ZW female only the left gonad becomes an ovary, while the right gonad regresses. Studies show that in the normal ZZ male embryo both $\mathrm{Z}$ chromosomes express DMRT1 in the developing testes and that there is no dosage compensation at this locus. Other Z-linked genes show equal levels of expression in both sexes and are dosage compensated (McQueen et al., 2001). As there is no sex chromatin in chicken it follows that dosage compensation of these genes does not occur by $\mathrm{Z}$ inactivation.

\section{W-Linked Female Sex Determination}

The small W chromosome of most birds, like the mammalian Y chromosome, has degenerated during evolution suggesting that it has developed a sex determining function for female differentiation. In the ZZW triploid intersex, the right gonad becomes a testis and the left gonad develops first into an ovotestis, at which time the bird appears phenotypically female (Lin et al., 1995). The ovarian part of the ovotestis then degenerates and the phenotype changes to male in the adult. This suggests that any $\mathrm{W}$-linked female determinant cannot be dominant. Only a small number of $\mathrm{W}$-linked genes have been reported and several of these (including ATP5A, CHD and $W P K C 1 / A S W$ ) have homologues on the $\mathrm{Z}$ chromosome, consistent with an origin of $\mathrm{Z}$ and $\mathrm{W}$ from a homomorphic pair of ancestral chromosomes (Smith and Sinclair, 2004). However, DMRT1 does not have a homologue on the W. One gene, female expressed transcript 1 (FET1), may be unique to the $\mathrm{W}$ and so represents a possible ovary-determinant. It is strongly expressed in the left gonad (developing ovary) of the ZW female embryo, consistent with this role. Another W-linked gene, WPKC1/ASW, has repeated copies on this chromosome and is also strongly expressed in the ovary of ZW female embryos. A single homologue of this gene, $Z P K C 1$, maps to the $\mathrm{Z}$ chromosome and is weakly expressed in embryos of both sexes. WPKC1 may promote ovarian development by repressing $Z P K C 1$ action, i.e. through a dominant negative effect on testis differentiation. An alternative theory implicates another W-linked gene (so far not characterised) that controls the expression of a male hypermethylated (MHM) region of the $\mathrm{Z}$ chromosome close to DMRT1. This region is undermethylated and transcribed on the single Z of ZW females, and methylated and repressed on both $\mathrm{Z}$ chromosomes of $\mathrm{ZZ}$ males (Teranishi et al., 2001). It is suggested that this mechanism serves to repress the expression of the adjacent DMRT1 gene in ZW females and hence promotes ovarian development. Thus, DMRT1 dosage and $\mathrm{W}$-linked factors may act in concert to determine avian sex. 


\section{Sex Determination in Reptiles, Fish and Amphibians}

Reptiles and fish seem to have evolved a confusing variety of mechanisms of sex determination and primary sex determining genes have not been identified (Schartl, 2004). All snakes investigated to date are like birds with female heterogamety and a ZZ/ZW system. In primitive snakes, such as the boid group, the $\mathrm{Z}$ and $\mathrm{W}$ chromosomes are homomorphic and cytologically indistinguishable, whereas in vipers and elapids the $\mathrm{W}$ is small, and in colubrid snakes the sex chromosomes are distinguishable only by the location of the centromere (Graves and Shetty, 2001). Turtles have karyotypes similar to birds with both macro- and microchromosomes and crocodiles have only macrochromosomes. Most turtles and all crocodiles have no sex chromosomes and sex is determined by the temperature at which the eggs incubate. In general, higher temperatures produce males in crocodiles and females in those turtles with temperature-dependent sex determination (TSD), although different temperature patterns are found in some species of both groups. It has been suggested that changes in temperature affect the expression of sex determining genes in the embryo during gonadal differentiation (Deeming and Ferguson, 1988). This hypothesis is supported by the observation that $D M R T 1$ expression in the genital ridge of turtle embryos is increased at the low temperatures that promote male rather than female differentiation (Kettlewell et al., 2000).

Several species of turtle have genetic sex determination (GSD), some with male and others with female heterogamety. Chelodina longicollis is an example of those species that have equal numbers of male and female offspring, irrespective of egg incubation temperature, and thus have genetic sex determination. Cross-species painting with chicken Z-specific paint reveals full length homology with the chromosome 5 pair in both male and female C. longicollis (Graves and Shetty, 2001). Chromosome 5 turns out not to be involved in primary sex determination in this turtle as comparative genomic hybridisation (CGH) between males and females reveals a heteromorphic microchromosome in males but not females (Ezaz et al., 2006). This finding is consistent with $\mathrm{X}$ and $\mathrm{Y}$ sex chromosomes and male heterogamety, and shows that in this species of turtle sex determination is different from the system that has evolved in birds. The primary testis-determining gene on the turtle $\mathrm{Y}$ has still to be identified.

Cross-species chromosome painting with chicken Zspecific paint probes has revealed apparently complete

Evolution of Sex Chromosomes and Sex

Determination homology with the homomorphic pair 6 in the red-eared slider, Trachemys scripta, and the Nile crocodile, Crocodylus niloticus, both of which have TSD (Kasai and Ferguson-Smith, unpublished). DMRT1 maps to chromosome 6 in both these species (Kasai and Ferguson-Smith, unpublished) and also to chromosome 6 in the Chinese soft-shelled turtle (Matsuda et al., 2005). Chromosome 6 in these reptiles thus seems to represent the ancestral pair of chromosomes from which the ZZ/ZW pair of chromosomes in birds has evolved.

The karyotypes of snakes show considerable rearrangement in comparison to those of turtles, crocodiles and birds that are highly conserved. The most striking difference concerns the snake $\mathrm{Z}$ and $\mathrm{W}$ sex chromosomes that are not homologous to the avian sex chromosomes and must have evolved independently from a different pair of ancestral autosomes. Orthologues of $\mathrm{Z}$ genes in the chicken, including DMRT1, map to chromosome 2 in both the Japanese four-striped rat snake, Elaphe quadrivirgata (Matsuda et al., 2005) and the Australian tiger snake, Notechis scutatus (Kasai and Ferguson-Smith, unpublished). Mapping studies indicate that the snake $\mathrm{Z}$ shares homology with chicken chromosome 2 and human chromosomes 7 and 10 . The primary sex determining switch and the role of DMRT1 in sex differentiation in snakes are not yet known. These genomic differences support the molecular phylogenetic data that suggest that snakes are more distantly related to birds and to other reptiles than was previously thought (Kumar and Hedges, 1998).

In lizards, both environmental and genetic factors are described in several species. TSD was first described for the lizard, Agama agama. In some lizards the female (ZW) is the heterogametic sex and in others it is the male (XY). A recent CGH study of the bearded dragon lizard, Pogona vitticeps, revealed ZZ/ZW microchromosomes (Ezaz et al., 2005) and thus another system different from snakes and birds. Incubation temperature has an influence on sex ratio in some lizard species with GSD.

The same variety of sex determining systems with both male and female heterogamety is found in fish and amphibians (Deeming and Ferguson, 1988). Among fish, sex determination in the medaka (Oryzias latipes) has been studied in some detail (Wittbrodt et al., 2002). The male (XY) is the heterogametic sex and, although the sex chromosomes are the second largest pair and morphologically indistinguishable, linkage studies reveal a sex determining cluster on a region of reduced recombination in the Y. SRY is absent but DMRT1 (termed DMY/ $D M R T 1 b Y$ ) maps to this cluster and also to a closely re- 
lated autosomal homologue, DMRT1a (Matsuda et al., 2002; Nanda et al., 2002). DMRT1bY is expressed exclusively in the adult testis, and mutations of the gene cause sex reversal. It is thus a good candidate for the primary sex determining signal in male embryos, although this has not been tested in transgenic studies (Kondo et al., 2006). However, molecular analysis in other fish species does not provide evidence of a Y-linked copy of DMRT1, and suggests that the Y-linked gene in medaka is a recent duplication (Volff et al., 2003). In zebrafish the absence of sex-linked markers has so far prevented an analysis of sex determination in this species. Only one DMRT1 gene locus has been identified in the draft sequence of the fugu genome.

Among amphibians, most information about sex determination relates to frogs and examples of species are described with heteromorphic XY and ZW sex chromosome systems, probably different from other vertebrate groups (Schmid et al., 2002a, b). Some species, including Rana rugosa, have homomorphic chromosomes and either male or female heterogamety (Ogata et al., 2003). In Rana rugosa, DMRT1 is expressed exclusively in the embryonic testes but not ovaries (Aoyama et al., 2003). However, DMRT1 is autosomal in this species and cannot be the primary signal for testis differentiation.

\section{Sex Determination in Monotremes}

Monotremes are egg-laying mammals that have a unique mixture of mammalian, avian and reptilian features. In addition to egg-laying and absence of teeth as in birds, the duck-billed platypus (Ornithorhynchus anatinus) has fur, primitive mammary glands without nipples, electro-sensors in its soft bill, and a pelvis similar in structure to the reptilian pelvis. There are two other extant monotremes, namely the short-beaked echid na (Tachyglossus aculeatus) and long-beaked echidna ( $\mathrm{Za}$ glossus bruijni). The ancestor of these three species is believed to have diverged from the mammalian lineage about 210 Mya and some 30 million years before the divergence of marsupials and placental mammals. The monotremes have been regarded therefore as more primitive than other mammalian species and are seen as forming a link between reptiles and placental mammals. As such, they may help to answer questions about sex determination and the origin of dosage compensation in mammals. Accurate chromosome identification is crucial for such studies, but karyotype analysis has been difficult in both platypus and echidna as both have un- paired chromosomes that form a multivalent chain at meiosis. Chromosome sorting and painting of platypus chromosomes has now revealed for the first time that the male platypus has a remarkable sex chromosome complement of five $\mathrm{X}$ chromosomes and five $\mathrm{Y}$ chromosomes (Rens et al., 2004). These form a chain of alternating X and $\mathrm{Y}$ chromosomes at meiosis, in which each of the ten components are linked together by pairing segments. The homology between each $\mathrm{X}$ and $\mathrm{Y}$ chromosome is revealed by chromosome-specific paint probes. The $\mathrm{X}$ and Y chromosomes undergo alternate segregation at meiosis so that sperm contain either five $\mathrm{X}$ or five $\mathrm{Y}$ chromosomes (Grützner et al., 2004). Females have five pairs of X chromosomes and do not form a chain at meiosis.

Preliminary gene mapping shows that $S R Y$ is absent in platypus as in birds, reptiles and fish. The large chromosome at one end of the chain, $\mathrm{X}_{1}$, has homology with the mammalian X. Comparative mapping with the human X chromosome (table 2) shows that many X-linked genes that map to the short arm of the human $\mathrm{X}$ are autosomal, as found in marsupials. However, as indicated in table 2, additional X-linked genes located in the long arm of the human $\mathrm{X}$ are also autosomal in platypus and map to chromosome 6 (Waters et al., 2005). These genes flank the $\mathrm{X}$-inactivation centre in placental mammals and, as they also map to the marsupial $\mathrm{X}$ and to chicken chromosome 4, their loss from the platypus $\mathrm{X}$ indicates an evolutionary change that has occurred in the monotreme lineage after the monotreme-metatherian/eutherian divergence. Being autosomal these genes do not require dosage compensation in monotremes, and this applies also to the $\mathrm{X}$-linked genes on the pairing region of platypus $\mathrm{X}_{1}$ which has homology with platypus $\mathrm{Y}_{1}$. As shown in table 2, some platypus genes homologous to genes on the long arm of the human X map to the short arm of the platypus $\mathrm{X}$ which is homologous to the long arm of $\mathrm{Y}_{1}$, and thus have similar dosage in both sexes without dosage compensation. Another surprising finding is that $D M R T 1$ is located on $\mathrm{X}_{5}$ in the platypus sex chromosome chain (Grützner et al., 2004). There is thus a double dose of DMRT1 in the female platypus and a single dose in the male. This is in contrast to mammals where two doses seem to be required for testis determination. In order to understand this paradox it will be necessary first to investigate DMRT1 expression in the developing gonads of platypus embryos, and then determine possible regulatory factors. It seems inconceivable that $D M R T 1$ is not involved. The location of DMRT1 on one of the platypus sex chromosomes is likely to be of significance, given its 
proven key role in sex differentiation in so many other vertebrate and invertebrate species.

Large regions of chromosomes $\mathrm{X}_{1}-\mathrm{X}_{5}$ in platypus are without homology to $Y_{1}-Y_{5}$, and some form of dosage compensation is to be expected to account for the malefemale difference. Several genes have been studied on platypus $\mathrm{X}_{1}$, and the androgen receptor and G6PD loci that are on the differential segment of $\mathrm{X}_{1}$ seem to have similar gene expression in both sexes (Grützner et al., 2003). There is some evidence that mechanisms of genome imprinting and $\mathrm{X}$-inactivation are absent in monotremes (Killian et al., 2000) and so it seems more likely that dosage compensation is achieved by up-regulation of $\mathrm{X}$-linked genes as in Drosophila.

The five $\mathrm{Y}$ chromosomes of the platypus deserve careful mapping and analysis to identify possible sex determinants within the substantial number of differential segments that have no homologous sequences in the female. These regions may also be expected to include loci important in male gametogenesis and fertility.

\section{Conclusion}

These observations on the evolution of sex determination in vertebrates indicate that the differentiation of a testis requires a gene cascade similar in all species but differing in the primary switch mechanism. $S R Y$ is the primary switch in almost all mammals, but the mole vole among rodents and the $S R Y$-negative XX male in humans show that a factor other than SRY must operate in these exceptional cases. As part of the sex differentiation cascade DMRT1 is expressed in higher dose in embryonic testes than in embryonic ovaries in all vertebrates (table 3). Temperature regulation of the expression of $D M R T 1$ may be the primary switch mechanism in turtles and crocodiles with TSD. In birds with female heterogamety, the most likely mechanism is dosage of the Zlinked $D M R T 1$ gene. In snakes, $D M R T 1$ is autosomal and the ZW sex determining system has evolved independently from the ZW system in birds, and from each of the different ZW systems in turtles and lizards (and probably also amphibians) with GSD. While several of the genes listed in table 1 are also involved in the early stages of testis determination, DMRT1 has a key role in all groups of vertebrates investigated to date, and it is perhaps not surprising that in birds, monotremes and one fish it is found in a sex chromosome. One awaits with interest the results of further studies in vertebrates that may reveal more about the mechanism of action of this interesting
Table 3. DMRT1 expression in the gonads of early embryos in representative vertebrate species

\begin{tabular}{llll}
\hline Species & Testis & Ovary & Reference \\
\hline Oryzias latipes & ++ & - & Volff et al., 2003 \\
Rana rugosa & ++ & - & Aoyama et al., 2003 \\
Trachemys scripta & ++ & + & Kettlewell et al., 2000 \\
Gallus domesticus & ++ & + & Raymond et al., 1999 \\
Macropus eugenii & ++ & + & Pask et al., 2003 \\
Mus musculus & ++ & + & Raymond et al., 1999 \\
\hline
\end{tabular}

gene, and also about the nature and evolution of the other primary signals for male differentiation in monotremes and non-mammalian vertebrates.

These findings also raise questions about the origin and evolution of sex determining systems in vertebrates. While the cascade of genes involved in testis differentiation is common to all vertebrates, the variety of sex chromosome systems and primary signals that initiate the same cascade suggest that these different mechanisms have evolved independently from a common ancestral condition. One can speculate that the ancestral condition could have been a form of TSD, as in some extant reptiles, and that different sex determination signals evolved on different chromosomes in separate lineages. Perhaps this depended on particular environmental factors, or on genes that conferred a reproductive advantage in one or the other sex. Advantageous genes would be maintained by natural selection on the various ancestral chromosomes that became $\mathrm{X}$ and $\mathrm{Z}$ types of sex chromosomes. The $\mathrm{Y}$ and $\mathrm{W}$ chromosome types would carry the primary determinants for testis and ovary respectively, taking over the role from a temperature-dependent system. The degeneration of the various $\mathrm{Y}$ and $\mathrm{W}$ chromosomes that has been observed among vertebrates, is the outcome of a system that has evolved to isolate the primary sex determinant from crossing-over during meiosis. Recombination is excluded from this region and so genes linked to the sex determinant accumulate mutations, lose function and are eventually deleted. Other genes essential for gametogenesis in testis or ovary are added in multiple copies to the $\mathrm{Y}$ or $\mathrm{W}$, being maintained by gene conversion rather than by meiotic recombination. Failure of an $\mathrm{XY}$ chromosome system, as is likely in SRY negative Ellobius species, leads to total loss of the $\mathrm{Y}$ and the evolution of a new GSD system in animals with either two X's or a single $\mathrm{X}$. The DMRT1bY system in medaka is another example of the comparatively recent occurrence of a new 
sex determining system within the past 10 million years. These hypotheses about the mechanisms involved in the evolution of independent sex determination systems will be refined once the nature of the primary determinants is resolved in vertebrate groups, such as the monotremes, where such information is currently lacking.

\section{Acknowledgement}

The author is grateful to Dr. Willem Rens and colleagues at the Cambridge Resource Centre for Comparative Genomics for valuable discussion during the preparation of this review.

\section{References}

Aoyama S, Shibata K, Tokunaga S, Takase M, Matsui K, Nakamura M: Expression of DMRT1 protein in developing and in sex reversed gonads of amphibians. Cytogenet $\mathrm{Ge}-$ nome Res 101:295-301 (2003).

Arakawa Y, Nishida-Umehara C, Matsuda Y, Sutou S, Suzuki H: X-chromosomal localization of mammalian Y-linked genes in two $\mathrm{XO}$ species of the Ryukyu spiny rat. Cytogenet Genome Res 99:303-309 (2002).

Calvari V, Bertini V, DeGrandi A, Peverali G, Zuffardi O, et al: A new submicroscopic deletion that refines the $9 p$ region for sex reversal. Genomics 65:203-212 (2000).

Deeming DC, Ferguson MWJ: Environmental regulation of sex determination in reptiles. Phil Trans R Soc London B 322:19-39 (1988).

Ezaz T, Quinn AE, Miura I, Sarre SD, Georges A, Graves JAM: The dragon lizard, Pogona vitticeps has ZZ/ZW microchromosomes. Chromosome Res 13:763-776 (2005).

Ezaz T, Valenzuela N, Grutzner F, Miura I, Georges A, et al: An XX/XY sex microchromosome system in a freshwater turtle, Chelodina longicollis (Testudines: Chelidae) with genetic sex determination. Chromosome Res 14:139-150 (2006)

Ferguson-Smith MA: Karyotype-phenotype correlations in gonadal dysgenesis and their bearing on the pathogenesis of malformations. J Med Genet 2:142-155 (1965).

Ferguson-Smith MA: X-Y chromosomal interchange in the aetiology of true hermaphroditism and of XX Klinefelter's syndrome. Lancet ii:475-476 (1966).

Ferguson-Smith MA: Genetic analysis by chromosome sorting and painting: phylogenetic and diagnostic applications. Eur J Hum Genet 5:253-265 (1997).

Ferguson-Smith MA, Affara NA: Accidental X-Y recombination and the aetiology of XX males and true hermaphrodites. Phil Trans R Soc Lond B 322:133-144 (1988).

Ferguson-Smith MA, Munro IB: Spermatogenesis in the presence of female nuclear sex. Scott Med J 3:39-42 (1958).

Ford CE, Jones KW, Polani PE, De Almeida JC, Briggs JH: A sex-chromosome anomaly in a case of gonadal dysgenesis (Turner's syndrome). Lancet i:711-713 (1959).
Glas R, Graves JAM, Toder R, Ferguson-Smith MA, O'Brien PCM: Cross-species chromosome painting between human and marsupial demonstrates the ancient region of the mammalian X. Mamm Genome 10:11151116 (1999)

Graves JAM: Sex and death in birds: a model of dosage compensation that predicts lethality of sex chromosome aneuploids. Cytogenet Genome Res 101:278-282 (2003).

Graves JAM, Shetty S: Sex from W to Z: evolution of vertebrate sex chromosomes and sex determining genes. J Exp Zool 290:449-462 (2001).

Graves JAM, Westerman M: Marsupial genetics and genomics. Trends Genet 18:517-521 (2002).

Grützner F, Deakin J, Rens W, El-Mogharbel N, Graves JAM: The monotreme genome: a patchwork of reptile, mammal and unique features? Comp Biochem Physiol Part A 136: 867-881 (2003).

Grützner F, Rens W, Tsend-Ayush E, EL-Mogharbel N, O'Brien PCM, et al: In the platypus a meiotic chain of ten sex chromosomes shares genes with the bird $\mathrm{Z}$ and mammal $\mathrm{X}$ chromosomes. Nature 432:913-917 (2004).

Jacobs PA, Ross A: Structural abnormalities of the $\mathrm{Y}$ chromosome in man. Nature 210:352354 (1966)

Jacobs PA, Strong JA: A case of human intersexuality having a possible XXY sex determining mechanism. Nature 183:302 (1959).

Just W, Rau W, Vogel W, Akhuerdian M, Fredga $\mathrm{K}$, et al: Absence of Sry in species of the vole Ellobius. Nat Genet 11:117-118 (1995).

Keitges E, Rivest M, Siniscalco M, Gartler SM: $\mathrm{X}$-linkage of steroid sulphatase in the mouse is evidence for a functional Y-linked allele. Nature 315:226-227 (1985)

Kettlewell JR, Raymond CS, Zarkower D: Temperature-dependent expression of turtle $D M R T 1$ prior to sexual differentiation. Genesis 26:174-178 (2000).

Killian JK, Byrd JC, Jirtle JV, Munday BL, Stoskopf MK, et al: M6P/IGF2R imprinting evolution in mammals. Mol Cell 5:707-716 (2000).

Kohn M, Kehrer-Sawatzki H, Vogel W, Graves JAM, Hameister H: Wide genome comparisons reveal the origins of the human X chromosome. Trends Genet 20:598-603 (2004).
Kondo M, Hornung U, Nanda I, Imai S, Sasaki T, et al: Genomic organization of the sex-determining and adjacent regions of the sex chromosomes of medaka. Genome Res 16:815826 (2006).

Kumar S, Hedges SB: A molecular timescale for vertebrate evolution. Nature 392:917-920 (1998).

Lahn BT, Page DC: Four evolutionary strata on the human X chromosome. Science 286 964-967 (1999).

Lin M, Thorne MH, Martin ICA, Sheldon BL, Jones RC: Development of the gonads in the triploid (ZZW and ZZZ) fowl, Gallus domesticus, and comparison with normal diploid mals (ZZ) and females (ZW). Reprod Fertil Dev 7:1185-1197 (1995).

Lyon M: Gene action in the mammalian X chromosome of the mouse (Mus musculus L.). Nature 190:372-373 (1961).

Matsuda M, Nagahama Y, Shinomiya A, Sato T, Matsuda C, et al: DMY is a Y-specific DM domain gene required for male development in the medaka fish. Nature 417:559-563 (2002)

Matsuda M, Nishida-Umehara C, Tarui H, Kuroiwa A, Yamada K, et al: Highly conserved linkage homology between birds and turtles: bird and turtle chromosomes are precise counterparts of each other. Chromosome Res 13:601-615 (2005).

McQueen HA, McBride D, Miele G, Bird AP, Clinton M: Dosage compensation in birds. Curr Biol 11:253-257 (2001).

Nanda I, Shan Z, Schartl M, Burt DW, Koehler $\mathrm{M}$, et al: 300 million years of conserved synteny between chicken $\mathrm{Z}$ and human chromosome 9. Nat Genet 21:258-259 (1999).

Nanda I, Tsend-Ajusch E, Shan Z, Grützner F, Schartl M, et al: Conserved synteny between the chicken $\mathrm{Z}$ sex chromosome and human chromosome 9 includes the male regulatory gene DMRT1: a comparative (re)view on avian sex determination. Cytogenet Cell Genet 89:67-78 (2000).

Nanda I, Kondo M, Hornung U, Asakawa S, Winkler C, et al: A duplicated copy of DMRT1 in the sex determining region of the $\mathrm{Y}$ chromosome of the medaka, Oryzias latipes. Proc Natl Acad Sci USA 99:11778-11783 (2002). 
Ogata M, Ohtani H, Igarashi T, Hasegawa Y, Miura I: Change of heterogametic sex from male to female in the frog. Genetics 164:613620 (2003).

Ohno S: Sex Chromosomes and Sex-linked Genes. (Springer-Verlag, Berlin 1967).

Palmer S, Perry J, Ashworth A: A contravention of Ohno's law in mice. Nat Genet 10:472-476 (1995).

Pask AJ, Behringer RR, Renfree MB: Expression of DMRT1 in the mammalian ovary and testis from marsupials to mice. Cytogenet Genome Res 101:229-236 (2003).

Raymond CS, Shamu CE, Shen MM, Seifert HJ, Hirsch B, et al: Evidence for evolutionary conservation of sex-determining genes. Nature 391:691-695 (1998).

Raymond CS, Kettlewell JR, Hirsch B, Bardwell VJ, Zarkower D: Expression of DMRT1 in the genital ridge of mouse and chicken embryos suggests a role in vertebrate sexual development. Dev Biol 215:208-220 (1999).

Reik W, Lewis A: Co-evolution of X-chromosome inactivation and imprinting in mammals. Nat Rev Genet 6:403-410 (2005).

Rens W, Grützner F, O'Brien PCM, Fairclough H, Graves JAM, Ferguson-Smith MA: Resolution and evolution of the duck-billed platypus karyotype with an $\mathrm{X}_{1} \mathrm{Y}_{1} \mathrm{X}_{2} \mathrm{Y}_{2} \mathrm{X}_{3} \mathrm{Y}_{3} \mathrm{X}_{4}$ $\mathrm{Y}_{4} \mathrm{X}_{5} \mathrm{Y}_{5}$ male sex chromosome constitution. Proc Natl Acad Sci USA 101:16257-16261 (2004).
Schartl M: Sex chromosome evolution in nonmammalian vertebrates. Curr Opin Genet Dev 14:634-641 (2004).

Schmid M, Feichtinger W, Steinlein C, Nanda I, Mais C, et al: Chromosome banding in Amphibia. XXII. Atypical Y chromosomes in Gastrotheca walkeri and Gastrotheca ovifera (Anura, Hylidae). Cytogenet Genome Res 96:228-238 (2002a).

Schmid M, Feichtinger W, Steinlein C, Rupprecht A, Haaf T, Kaiser K: Chromosome banding in Amphibia. XXIII. Giant W sex chromosomes and extremely small genomes in Eleutherodactylus euphronides and Eleutherodactylus shrevei (Anura, Leptodactilidae). Cytogenet Genome Res 97:81-94 (2002b).

Shetty S, Griffin DK, Graves JAM: Comparative painting reveals strong homology over 80 million years of bird evolution. Chromosome Res 7:289-295 (1999).

Sinclair AH, Berta P, Palmer MS, Hawkins JR, Griffiths BL, et al: A gene from the human sex determining region encodes a protein with homology to a conserved DNA-binding motif. Nature 346:240-244 (1990).
Smith CA, Sinclair AH: Sex determination: insights from the chicken. BioEssays 26:120132 (2004)

Spencer JA, Sinclair AH, Watson JM, Graves JA: Genes on the short arm of the human X chromosome are not shared with the marsupial X. Genomics 11:339-345 (1991).

Teranishi M, Shimada Y, Hori T, Nakabayashi O Kikuchi T, et al: Transcripts of the MHM regions on the chicken $\mathrm{Z}$ chromosome accumulate as non-coding RNA in the nucleus of female cells and adjacent to the DMRT1 locus. Chromosome Res 9:147-165 (2001).

Volff J-N, Kondo M, Schartl M: Medaka $d m Y /$ $d m r t 1 Y$ is not the universal primary sexdetermining gene in fish. Trends Genet 19: 196-199 (2003).

Waters PD, Delbridge ML, Deakin JE, ElMogharbel N, Kirby PJ, et al: Autosomal location of genes from the conserved mammalian X in the platypus (Ornithorhynchus anatinus): implications for mammalian sex chromosome evolution. Chromosome Res 13:401-410 (2005)

Wittbrodt J, Shima A, Schartl M: Medaka - a model organism from the Far East. Nat Rev Genet 3:53-64 (2002). 